\title{
Coupling for Power Line Communication: A Survey
}

\author{
Luis Guilherme da S. Costa, Antonio Carlos M. de Queiroz, Bamidele Adebisi, Vinicius L. R. da Costa, and \\ Moises V. Ribeiro
}

\begin{abstract}
The advent of power line communication (PLC) for smart grids, vehicular communications, internet of things and data network access has recently gained ample interest in industry and academia. Due to the characteristics of electric power grids and regulatory constraints, the effectiveness of coupling between the power line and PLC transceivers has become a very important issue. Coupling devices used to inject or extract data communication signals into or from power lines are very important components of a PLC system. There is, however, an obvious gap in the literature for a detailed review of existing PLC couplers. In this paper, we present a comprehensive review of couplers, which are required for narrowband and broadband PLC transceivers. Prevailing issues that protract the design of couplers and consequently subtended the inventions of different types of couplers are clearly described. We also provide a useful classification of PLC couplers based on the type of physical couplings, voltage levels, frequency bandwidth, propagation modes and a number of connections. This survey will guide researchers, as well as designers alike, into a quicker resourcing when studying coupling in narrowband and broadband PLC systems.
\end{abstract}

Index Terms-Power line communication, coupling, impedance matching, electric power system.

\section{INTRODUCTION}

The use of power cables for data communication purposes, known as Power Line Communication (PLC), dated back to the early 1910s when Major George Squier of US Army demonstrated the transmission of analog voice signals (multiple telephony channels) over a pair of power cables to support the operation of distribution power systems by electric utilities. At that time, this type of analog data transmission was called wired wireless [1]. The period from 1910 to 1930 was characterized by the introduction of some technologies which involve the transmission of telephone signals through power cables.

The PLC has since moved from the little-known technology in the 30 s to a competitive technology of the 21 st century. As a consequence, coupling issues have become more relevant. Essentially, couplers are devices which inject data signals into

The Ad Hoc Associate Editor coordinating the review of this manuscript and approving it for publication was Prof. Renato Machado.

L. G. S. Costa (luis.guilherme@engenharia.ufjf.br) is with the Electrical Engineering Department, Federal University of Juiz de Fora (UFJF), Brazil.

A. C. M. de Queiroz (acmq@coe.ufrj.br) is with the COPPE, Electrical Engineering Program, Federal University of Rio de Janeiro, Rio de Janeiro, Brazil.

B. Adebisi (b.adebisi@mmu.ac.uk) is with the School of Engineering, Manchester Metropolitan University, Manchester, UK.

Vinicius L. R. da Costa (vinicius.lagrota@engenharia.ufjf.br) is with the Electrical Engineering Department, Federal University of Juiz de Fora (UFJF), Brazil.

M. V. Ribeiro (mribeiro@ieee.org) is with the Electrical Engineering Department, Federal University of Juiz de Fora (UFJF), and Smarti9 Ltd., Brazil.

Digital Object Identifier: 10.14209/jcis.2017.2 electric power cables. These power cables could be alternating current (AC) or direct current (DC) power lines and the signals of PLC transceivers are subsequently coupled to them via a coupling circuit. In the case of power lines used to transmit $\mathrm{AC}$ power, the coupling circuit has also to filter out the $\mathrm{AC}$ mains signal. On the other hand, the coupling circuit simply has to block the DC mains voltage of the DC electric power grids.

During the late 1970s and early 1980s, new investigations to characterize electric power grids as a medium for data communication showed a higher potential in the range of frequencies between $5 \mathrm{kHz}$ and $500 \mathrm{kHz}$ [2]. Some years later, it was demonstrated that, given some constraints (transmission power and distance), the frequency range from $9 \mathrm{kHz}$ to $30 \mathrm{MHz}$ could be used [3]. Currently, there are researches and development efforts focusing on the use of electric power grids in the frequency band between 0 and $500 \mathrm{MHz}$.

Moreover, PLC systems can be distinguished based on two classes of frequencies: narrowband and broadband. Usually, narrowband PLC refers to data communication over the frequency band between 0 and $500 \mathrm{kHz}$ [4]. On the other hand, broadband PLC covers the frequency band between $1.7 \mathrm{MHz}$ and $500 \mathrm{MHz}$ and high-speed data rate [5]-[7].

It is important to mention that the main focus on PLC technologies is to provide data communication over AC and DC electric power grids. The use of PLC technologies over the DC bus in distributed energy generation, airplanes, automobiles and trains reduce wiring complexity, weight, space requirement and ultimately installation cost of telecommunication infrastructures. Thus, the PLC technologies eliminate the need for extra wires dedicated for data communication, which, for instance, reduces the weight of vehicle and prevent problems when passing wires through dashboard and instrumentation panels. They also decrease cost and complexity of cable connectors and raise the benefit-cost ratio to any new device requiring extra wiring [8]. Therefore, the investigation of low-cost couplings is of utmost importance for such kind of environments.

Electric power systems have the advantage of being a ubiquitous infrastructure [9]. Also, no changes in the installed power cables infrastructure are necessary to implement the PLC system. As a result, the payback can be quicker with reduced capital expenditures. PLC networks can be used as a backbone of data communication networks and considered as a technology for replacing, completing, serving or interconnecting with other data networks. The cost of PLC systems is moderate for long-term purposes, especially when compared to the conventional data network systems. For example, the replacements of these power cables are not comparable in cost with the replacement of the conventional data communication 
system devices such as cables, switches and outlets [10].

Reviewing the literature about PLC technologies, measurement [11], [12], characterization [13] and modeling, as well as the development of techniques to improve the performance of PLC systems at the physical and link layers [14]-[17], there are a number of technical contributions providing understanding into how PLC systems work and pointing out challenging issues related to the advancement of the PLC technology. Although there is a plethora of literature on the general PLC systems, a similar volume is currently not available for coupling techniques in PLC systems. Hence, the relevance of this survey.

In the literature, antenna coupling was the first type of coupling used with the electric power grid due to its simplicity, cost and easy installation. However, impedance mismatching with the power cables, which reduces the transfer of energy between the power cable and the antenna, motivated investigations which resulted in the introduction of capacitive couplers [1], [18]. It is interesting to note that one century later, researchers are reinvestigating the use of antennas to introduce the so-called PLC wireless system [19], [20]. In the sequel, inductive coupling was introduced. Due to the characteristics of inductive couplings such as electromagnetic isolation between the power cable and the PLC transceiver, they have been successfully applied in Medium-Voltage-based (MV) PLC systems since it is possible to install them to the live power line. On the other hand, their usage in electric power systems Low-Voltage (LV) was not widely adopted due to the high insertion loss. For LV levels, capacitive coupling is common due to its low cost and simplicity. Most recently, resistive PLC couplers have gained some interest due to their low cost and simple circuitry features [21].

Above all, coupling is a very important issue in PLC systems. Since the dynamism of electric power systems at High Voltage (HV), MV and LV levels and, as a consequence, the lack of perfect impedance matching at the point of connection with the PLC transceiver both in the time and frequency domains may result in remarkable insertion loss and signal distortion [22].

In this context, this work aims to present a comprehensive review on coupling for PLC systems. With this in mind, we first discuss important issues related to the design of PLC couplers, such as printed circuit board (PCB) design, insertion loss and return loss, galvanic isolation, impedance matching, analog filtering, protection and performance due to the type of components, costs and complexities. Second, we present a classification of couplings which considers the type of physical coupling (capacitive, inductive, resistive and antenna), the voltage levels (HV, MV and LV), the type of voltage (AC or DC), the frequency band (narrowband and broadband), the type of signal propagation (common mode (CM) or differential mode (DM)) and the number of connections. Additionally, we highlight future tendencies and critical issues which must be pursued to introduce PLC couplers which will comply with the constraints imposed by the electric power systems and fulfill the needs and demands associated with the novel generation of PLC systems.

The remaining parts of this work are organized as follows:
Section II briefly and concisely addresses the main issues for designing PLC couplers; Section III discusses a classification of PLC couplers based on a comprehensive review of this subject; Section IV highlights open problems and future trends for advancing the design of PLC coupling devices; and, finally, concluding remarks are stated in Section V.

\section{Design of A PLC Coupler: Important Issues}

The coupling of PLC transceivers with electric power grids is considered to be a difficult task to be accomplished mainly because the access impedance (impedance at the point of connection) changes with frequency and time due to the dynamism of loads connected to electric power grids. Also, the low impedance of power cables, which is desired for reducing technical losses associated with the energy delivery, yields a problem because the usual coupling devices are designed to have access impedance equal to $50 \Omega$ or $75 \Omega$.

The design of a PLC coupler considering impedance matching, flat frequency response to minimize distortions, efficient electrical protection against transients and low cost have been the major challenges in recent years. To carry out such design, several issues must be addressed in order to come up with a PLC coupler which may be useful for the target application. In this context, it is necessary to understand all main issues which must be taken into account to correctly design a PCB, such as measuring $S$ scattering parameters for quantifying insertion and return losses, providing galvanic isolation, dealing with impedance matching, designing the analog filters with passive components and choosing the appropriate electric protection scheme. These relevant issues are briefly described in the following subsections.

\section{A. Printed circuit board design}

The electrical characteristics of the PCB used to physically assemble the components of the coupling device significantly impact on the coupling performance [23]. In [24], the author showed that the transmission line properties of signal traces in the PCB considerably changes when multiple circuits or components are connected to a given signal trace. Characteristics such as impedance mismatching and skin effect usually result in an increase of the conductor impedance as the frequency rises.

Moreover, as frequency increases, parasitic elements may impact the design of the PCB of PLC couplers and their modeling for circuit simulation and design becomes relevant [25]. In a high-frequency circuit, it is simple to visualize how a long thin track of a PCB will behave as an inductor, a large pad over a ground plan will behave as a capacitor and performance degradation may occur due to the crosstalk among tracks [26]. In [27], the authors discussed the impedance mismatching in the connections among tracks, capacitors, pathways, stubs and welds.

In [28], high-frequency transmission line effects were studied and a fundamental concept called electrically long trace was defined. If a distance from transmitter to receiver exceeds $\lambda / 20$, where $\lambda$ is the wavelength in meters, or terminates the transmission line in its characteristic impedance when the 
one-way propagation delay of the PCB track is equal to or greater than one-half the applied signal rise/fall time, then the PCB should be treated as a transmission line and a routing topology should be created to match the trace impedance of a PCB. To match impedance line in a PCB, several techniques are available for designing a transmission line structure. There are two techniques which are most frequently used to control trace impedance, stripline and microstrip. In stripline, the transmission lines exist on internal routing layers and require a minimum of 3 board layers ( 2 ground planes and a routing layer). The insulating material of the substrate forms a dielectric. The width of the strip, the thickness of the substrate and the relative permittivity of the substrate determine the characteristic impedance of the strip, which is a transmission line. The use of microstrips is a popular method used to provide controlled trace impedance when microwave frequency is taken into account. Microstrip lines are exposed to both air and dielectric material referenced to the planar structure. Fig. 1 shows a microstrip transmission line structure, where $T$ is the thickness of the track, $W$ is the width of the track and $h$ is the distance between signal track and the reference plane.

The stripline technique has the advantage of greater isolation of transmission lines and bandwidth, resulting in lower radiation loss. Although better isolation and good electromagnetic shielding can be achieved, the stripline technique has the disadvantage of complexity and cost in its fabrication, as tuning, or troubleshooting is complex and stripline trace width is smaller compared to a microstrip line of same impedance and height. Microstrip has the advantage of less dielectric losses (when using identical materials), being cheaper and presenting easier debugging due to the location of traces on the top and bottom layers [29].

Characteristic impedance $Z_{0}$ of microstrip is also a function of the ratio of the height to the width $W / h$ (and the ratio of width to height $h / W$ ) of the transmission line. The characteristic impedance $Z_{0}$ of a microstrip is calculated by [30]

$Z_{0}=\left(\frac{87}{\sqrt{\varepsilon_{r}+1.41}}\right) \ln \left(\frac{5.98 h}{0.8 W+T}\right) \Omega \quad$ for $\quad 15<\mathrm{W}<25$ mils

or

$Z_{0}=\left(\frac{79}{\sqrt{\varepsilon_{r}+1.41}}\right) \ln \left(\frac{5.98 h}{0.8 W+T}\right) \Omega \quad$ for $\quad 5<\mathrm{W}<15$ mils,

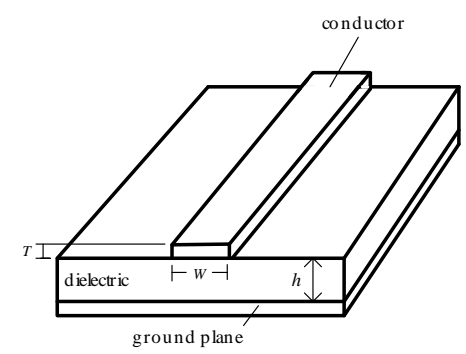

Fig. 1: A PCB microstrip transmission line.

where $\varepsilon_{r}$ denotes the dielectric constant of the PCB material.

To implement a ground plane, one side of a double-sided $\mathrm{PCB}$ is made of continuous copper plate and used as ground.
The theory behind it is that the larger the amount of metal, the lower the attained resistance. An analytical method for characterizing the impedance behavior of the ground planes in the frequency domain is presented in [31]. The importance of maintaining a large area of low-impedance ground plane is critical to all analog circuits today. The ground plane not only acts as a low impedance return path for decoupling high-frequency currents but also minimizes electromagnetic interference (EMI) emissions [32].

To make full use of PLC couplers for data communication purposes or in order to analyze the impedance matching, it is important to know how signals propagate on a PCB to obtain low insertion loss in a microstrip transmission line.

\section{B. Insertion loss and return loss}

Assuming that the two ports use the same reference impedance the insertion loss $\left(I_{L}\right)$ is the magnitude of the $S_{21}$ scattering parameter, given (in $\mathrm{dB}$ ) by:

$$
I_{L}(d B)=-20 \log _{10}\left|S_{21}\right|,
$$

where $S_{21}$ represents the voltage gain from Port 1 to Port 2 [33], which is often used to show the frequency response of passive circuit which is supposed to be linear and timeinvariant [34]. The Insertion Loss is defined as the drop in power as a signal enters in the two port networks. This value not only includes the reflected incoming signal but also the attenuation of the component and it is the ratio between output power and input power [35].

The return loss $\left(R_{L}\right)$ is the magnitude of the $S_{11}$ scattering parameter, which represents the reflection coefficient, because it describes what fraction of an incident wave is reflected back to the input port. In other words, the $S_{11}$ scattering parameter is a measure of how close the source and input impedances are matched. The $R_{L}$ given (in $\mathrm{dB}$ ) by

$$
R_{L}(d B)=-20 \log _{10}\left|S_{11}\right| .
$$

In order to measure $S_{12}$ and $S_{11}$ scattering parameters, the impedances of a vector network analyzer (VNA) are matched to $50 \Omega$, the passive PLC coupler is considered as a two-port network and, as a consequence, we characterize it through its scattering matrix, as shown in Fig. 2 [36]. In Fig. 2, the $Z_{\text {network }}$ represents the impedance of the electric power network at the connection point, $\Gamma_{\text {in }}$ and $\Gamma_{\text {out }}$ represent the reflection coefficients of PLC coupler and $Z_{\text {network }}$, respectively. Finally, $Z_{2}$ represents the impedance of port 2 - 2' of the PLC coupler. With knowledge of the scattering parameters, we can completely characterize PLC coupler and precisely quantify the distortion introduced by the PLC coupler in the transmitted signal.

\section{Galvanic isolation}

Galvanic isolation is usually necessary when two or more electric circuits are connected, but their grounds may be at different potentials. Also, it is an effective approach for breaking ground loops by preventing undesirable current flowing between two distinct circuits sharing a ground conductor. Moreover, it protects transceiver circuits and people from 


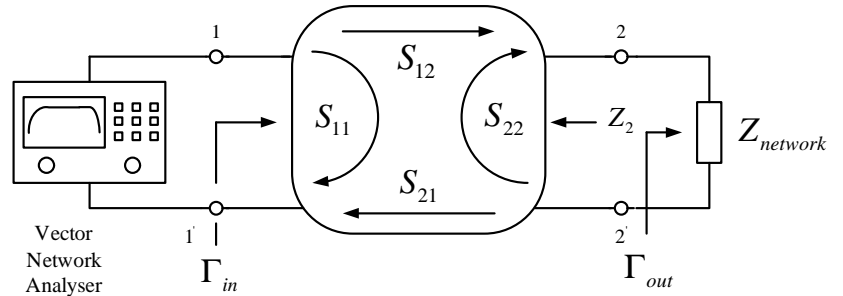

Fig. 2: Measurement of scattering parameters.

shocks and short circuits. Regarding the PLC coupler, a radio frequency $(\mathrm{RF})$ transformer is normally responsible for the galvanic isolation [37] between the transceiver and the power line. Also, as discussed in [38]-[40], it can be applied to impedance matching purposes through the transformation ratio 1: $N$ between the primary and secondary windings of the transformer, where $N$ denotes the turns ratio of secondary to the primary windings.

In [41], it was shown that an RF transformer has some nonideal characteristic properties which must be observed, such as the maximum magnetic flux density in the core to avoid nonlinear effects, frequency band specification, impedance levels/winding ratio, maximum voltage levels, maximum power and currents, skin effect, number of strands, number of turns, leakage inductance, enlarging of leakage inductance, magnetizing inductance and distortion of signal in secondary winding.

In [37], a model of an RF transformer was proposed and analyzed by Kazimierczuk. Fig. 3 depicts such model. It consists of ideal transformer, magnetizing inductance $L_{M}$, core-loss shunt resistance $R_{C}$, leakage inductances $L_{l P}$ and $L_{l S}$, winding resistances $R_{P}$ and $R_{S}$ and stray capacitances $C_{P}$ and $C_{S}$. The stray capacitances model the effect of turn-to-turn capacitance, layer-to-layer capacitance, windingto-winding capacitance, winding-to-core capacitance, windingto-shield capacitance, core-to-shield capacitance and the capacitance between the outer winding and surrounding circuitry [42]-[45].

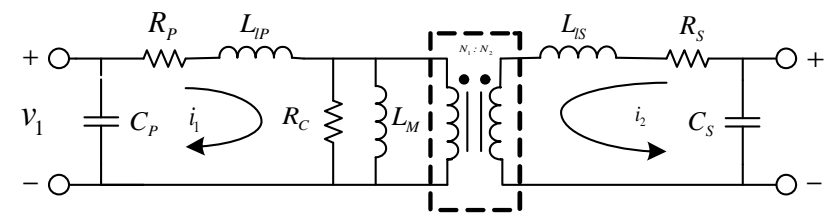

Fig. 3: Model of RF Transformer.

In order to determine the bandwidth of an RF transformer, low frequency and high frequency are analyzed. For a low frequency of an RF transformer, the magnetizing impedance is calculated by $j \omega L_{m} \backslash \backslash R_{C}$. The values of $L_{M}$ and $R_{C}$ are determined by the core material and the cut-off frequency can be calculated by the core material with high relative permeability. High-frequency cut-off is attributed to intrawinding capacitance $\left(C_{P}, C_{S}\right)$ and series leakage inductance $\left(L_{l P}, L_{l S}\right)$, core and conductor losses $\left(R_{P}, R_{S}\right)$. To achieve a high value of frequency, the stray capacitance $\left(C_{P}, C_{S}\right)$ and leakage inductance $\left(L_{l P}, L_{l S}\right)$ should be reduced.

\section{Impedance matching}

The impedance matching between the PLC coupler and the power cable at the connection point is a challenging task to be accomplished due to the time-frequency varying nature of the loads connected to electric power grids [46]-[48]. Due to the wide range of values that the impedance at the connector point (access impedance) can assume when the frequency and time vary [49]-[51], it definitely must be brought to the center of discussion when designing a PLC coupler. In fact, the lack of impedance matching may considerably reduce the transmission power [52]-[58] even though impedance matching is ensured within the PCB of the PLC coupler.

Impedance matching techniques for narrowband PLC couplers were proposed in [58], [59]. The impedance matching technique based on the change of the tap of an RF transformer was outlined in [60]. In [61] the authors described a technique for measuring the impedance at the connection point of an electric power grid to feed a microcontroller, which is capable of switching a bank of capacitors [62] aiming to eliminate the reactive component of the access impedance. The complexity of this technique increases along with the number of capacitors in the bank. Moreover, the variation step of the impedance depends on the number of branches of the bank of capacitors. Then, [58], [63], [64] proposed a new circuit, which is capable of adapting the impedance, changing the series inductance and the turns ratio of the RF transformer. Transformerless coupling circuits and impedance adaptation for narrowband PLC, with a view of simplifying the design and minimizing costs of a PLC transceiver, were discussed in [65], [66].

Impedance matching techniques for broadband PLC were discussed in [67], [68]. These works addressed an optimization method to calculate the values of the parameters of $5^{\text {th }}$ order band-pass filters for matching the access impedance. Moreover, [58] focused on another design method which is capable of offering an optimization procedure to synthesize broadband impedance matching circuits for the equalization of the power transfer gain in a wide frequency band. The method is based on a parametric representation of the driving impedance of the broadband impedance matching circuit, which is optimized by means of a meta particle swarm optimization method [69].

Impedance matching to improve the maximum power transfer of transmission/reception of signal into/from power line cable becomes an important and challenging issue. Different designs of impedance matching circuit have been studied in the literature. Frequency and time dependencies of access impedance, which renders adaptive matching circuits to be developed, are the major challenge of PLC coupler.

\section{E. Analog filtering}

In a typical PLC coupler design, analog filtering is performed by a cascade of a high-pass filter and a low-pass filter, in this order, and their combination results in a passband filter. The design of an equivalent passband filter is not recommended because the purposes of designing the high-pass and low-pass filters are totally different. In fact, the former aims to block the high power mains signal [70], while the latter limits the upper edge of frequency bandwidth to comply with 
the analog-to-digital conversion [71]. Fig. 4 shows a typical capacitive single-input and single-output (SISO) PLC coupler, where $T_{1}$ is an RF transformer, $C_{B L O C K}$ is a high-pass filter and $L_{1}, L_{2}, C_{1}$ up to $C_{5}$ constitute a low-pass filter.

The design of the high-pass filter is very simple because it is realized with a capacitor with a reasonable working voltage and a self-resonant frequency higher than the maximum mains frequency. On the order hand, the low-pass filter is more sophisticated and can be realized with a Butterworth, Chebyshev or an elliptic filter. Usually, the choice is in favor of an elliptic filter due to the sharpness of its frequency response [72]-[75]. However, a careful analysis of the lowpass filter must be carried out when multi-carrier scheme based on Discrete Fourier transform (DFT) is used because it can increase the effective delay spread of the channel between the digital-analog converter (DAC) of the transmitter and the analog-digital converter (ADC) of the receiver. Moreover, a practical approach for reducing the order of a low-pass filter is to concatenate a low-order high-pass filter (less selective) with a $2^{\text {nd }}$ or $3^{\text {rd }}$ order notch filter with notch frequency located in the stopband. Additionally, the analog filter can be used to mitigate interference from radio signals (AM, FM and TV) [76], [77]. The order of the analog filter can be determined by the designer according to the desired selectivity, keeping in mind that the insertion loss increases along with it. Therefore, a rule of thumbs is to keep the analog filter order below or equal to six.

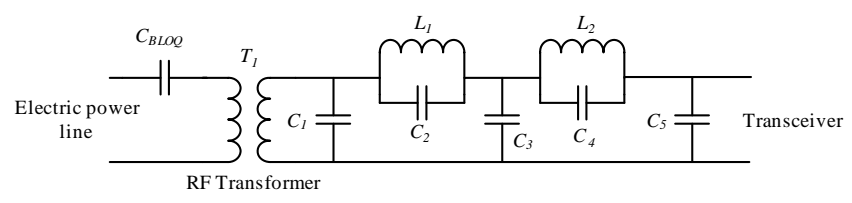

Fig. 4: Circuit of a capacitive PLC coupler.

\section{F. Electric protection}

All telecommunication technologies are bound to comply with electromagnetic compatibility (EMC) standards, whose requirements depend on the environment [78], [79]. The PLC coupling circuit, which is physically connected to power cables, is occasionally subjected to significant high voltage and current transients and, as a consequence, damages to the circuits of the PLC transceiver may happen [80], [81]. For instance, in $\mathrm{HV}$ and $\mathrm{MV}$, a strong current pulse of short rise time is created when a distribution transformer fails due to a short circuit or when power cables fall to the ground (high impedance faults) or touch each other. Similarly, a lightning strike near the power cable yields a traveling wave that may destroy the PLC transceiver.

With this in mind, surge protective devices (SPD) attempt to limit the voltage supplied to an electric device by either blocking or shorting to ground any unwanted voltages above a safe threshold. An SPD must simultaneously provide low insertion loss in all operating frequency band and adequate surge protection in all ports of the PLC coupler. The SPD must not interfere with the frequency spectrum of the transmitted signal. Typical SPD used in PLC couplers are metal oxide varistor (MOV), Zener diodes, high-speed switching diodes and gas discharge tubes (GDT).

In [82], a surge protection circuit for HV-based PLC systems was proposed aiming to achieve protection against an induced surge in one conductor of the transmission line, to offer protection against a common mode surge induced in both conductors of the transmission line and to yield protection against the potential rise of the earth conductor during an occurrence of high surge current through the earth conductor.

The authors of [83] analyzed the influence of capacitance of an MOV on the insertion loss of a PLC coupler. Although, the capacitance characteristics of an MOV and diodes, such as Zener and high-speed switching diodes [52], may cause distortion in the frequency response $\left(S_{12}\right.$ or $S_{21}$ scattering parameter) of PLC couplers, their usage is widely adopted because the cost-benefit is the best available. In order to avoid the disturbing effect of MOVs, GDTs can replace them in PLC couplers designed to operate at frequencies above $35 \mathrm{MHz}$. Regarding the frequency band between 1.7 and $100 \mathrm{MHz}$, Fig. 5 shows the effect of an MOV and Zener diode working together in concatenated way on the frequency response magnitude of a PLC coupler in comparison with a GDT. It is important to mention that the majority of studies and simulations of SPD effect covers the frequencies up to 30 $\mathrm{MHz}$ [2]. However, nothing has been discussed in the literature about its protection efficiency capabilities and its impact on the signal distortion at the frequencies higher than $30 \mathrm{MHz}$. Furthermore, the use of transient voltage surge protection (TVSP) [84] can also be investigated to replace Zener diodes or high-speed switching diodes for frequencies above $35 \mathrm{MHz}$ in PLC coupler.

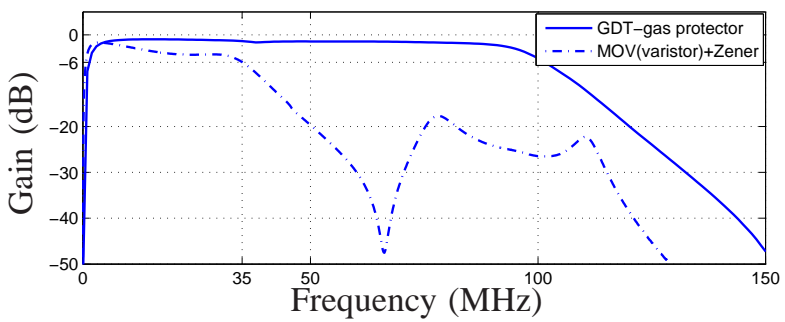

Fig. 5: Distortion of frequency response magnitude of a capacitive and low voltage PLC coupler in the frequency band $1.7-100 \mathrm{MHz}$ due to the use of different types of protective circuit.

Fig. 6 shows a typical protection for inductive and capacitive PLC coupler circuits. The capacitive coupler requires the use of MOV, fuse and Zener diodes to protect the transceiver circuit. The inductive coupler does the same by exploiting the limitations of the maximum magnetic flux density of the ferrite core to avoid spikes of current in the secondary windings and by concatenating high-speed switching diodes at the secondary windings of the ferrite core to limit the voltage. The use of MOV [85] and diodes for protection purposes needs to be analyzed based on the operating frequency of the PLC system and the voltage level of the mains signal in the electric power line. 


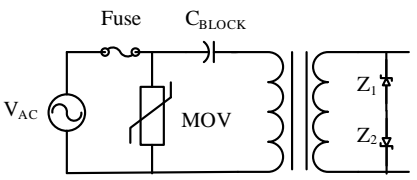

(a)

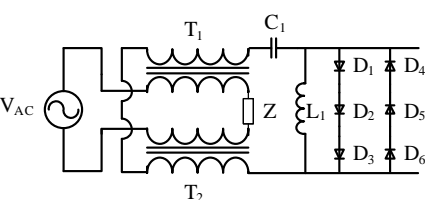

(b)
Fig. 6: Typical protection circuit for PLC coupler. (a) Capacitive coupler and (b) Inductive Coupler.

\section{G. Components}

Due to cost and size, the components used in a PLC coupler circuit are passive (resistors, capacitors, transformers, and inductors). Therefore, particular attention must be paid to the self-resonant frequency of inductors and capacitors as well as to the frequency response of the transformer. Additionally, the behavior of other components due to the chosen operating frequency of the PLC system needs to be carefully taken into account, especially at high frequencies [86], as the skin effect, i.e. the increase in the apparent resistance of a wire along with the frequency, becomes more relevant. If a voltage is maintained at a constant DC level, current flow will be uniform throughout the area of the wire. However, as the frequency increases, the magnetic field near the center of the wire increases the local reactance. The charge carriers then move towards the edge of the wire, decreasing the effective area and increasing the apparent resistance of the inductor winding [87], [88], capacitor [89], and resistor.

In fact, at high frequencies, the physical length, width and height of components, the properties of the conductors and dielectrics, as well as the types of electrodes for attachment to external circuits have influence on the performance of a PLC coupler circuit. Fig. 7 shows high-frequency lumped circuit models for a resistor, a capacitor and an inductor. Note that the additional capacitance due to the attached electrodes is usually included in the nominal value of the capacitance. Despite the parasitic resistances and reactances, attaching devices on a PCB can introduce significant additional capacitance to the body of the component [42]. This capacitance and the added inductance of the pads and traces, which are used to attach a component and are typically considered part of the circuit, affect the frequency response and impedance matching of a PLC coupler. In the design of PLC the coupler for broadband data communication, it is important to take it into account.

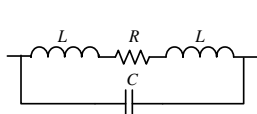

(a)

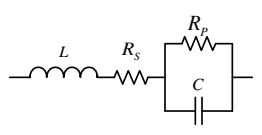

(b)

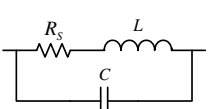

(c)
Fig. 7: Equivalent circuit for RF model. (a) Resistor, (b) Capacitor and (c) Inductor.

\section{H. Cost and complexity}

The main advantages of the PLC technology are the use of electric power system infrastructures for costs and expenditures reduction and the easy deployment of new telecommu- nication networks over such infrastructure [90]. Regarding HV and MV PLC couplers, some care must be taken into account due to the complexity of the coupler construction, which may increase costs due to the isolation need at these voltages levels. Moreover, PLC coupler designers must pay particular attention to the external environment which is subject to rain, snow and lightning on the power cable [91]. Due to these constraints and environment issues, the cost of the capacitive coupler is very high and high at $\mathrm{HV}$ and MV, respectively. Due to saturation of magnetic flux inside the core and the cost to handle it, inductive coupling is only used at MV and LV. Moreover, at LV, PLC couplers are constructed at a relatively low cost. Usually, a PLC capacitive coupler requires low complexity and cost since the components are available commercially. In [66], a PLC coupler without transformer was proposed for eliminating this costly component, while maintaining impedance matching with the electric power grids. Overall, complexity and cost of a PLC coupler increase along with voltage levels and bandwidth. Also, they increase when perfect impedance matching is mandatory.

\section{ClassificAtion OF PLC COUPLERS}

In general, PLC couplers can be grouped according to different criteria. In this sense, this work outlines a classification based on the type of physical couplings, voltage levels, frequency bandwidths, propagation modes and numbers of connections.

\section{A. Based on the type of physical connection}

Based on the type of physical connection, the coupling can be capacitive, inductive, resistive and by an antenna. Note that the antenna could be seen as a kind of inductive coupling, but we prefer to keep it separate because its mechanical construction is totally different.

1) Capacitive: Capacitive coupling is the serial insertion of one or more capacitors in direct contact with the power cable. It offers low impedance path to the high-frequency components of the power line signal and, at the same time, it blocks the mains signal by presenting a high impedance to it. Among the four types of PLC couplers, it has the lowest insertion loss, thus ensuring the highest power transfer [92]. As shown in Fig. 8, there are two types of capacitive coupler: (a) one with a transformer to provide galvanic isolation $\left(T_{1}\right)$ and to limit transients based on the core saturation; (b) and one without transformer, which is a low-cost option with the disadvantage of not providing galvanic isolation. While the use of capacitive coupling with transformer is strongly recommended in $\mathrm{AC}$ electric circuits, mainly when the voltage amplitude of the mains signal increases, the use of capacitive coupler may also be useful for DC buses [93], [94] in which the voltage level is low, such as in vehicle [95], ships and spacecraft environments. In [96], the author proposed a capacitive coupler to operate in low-voltage DC electric power grids when the frequency bandwidth is between $1 \mathrm{MHz}$ and $30 \mathrm{MHz}$ and the voltage level of mains signal is up to $750 V_{D C}$. 


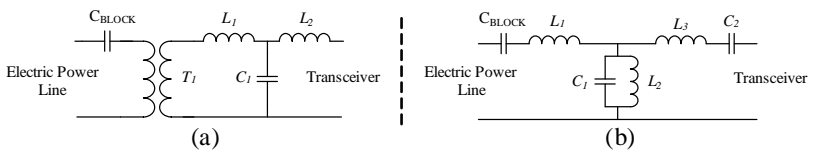

Fig. 8: Equivalent circuit for a capacitive PLC coupler. (a) Coupling with galvanic isolation and (b) Transformerless coupling.

2) Inductive: The inductive PLC coupler can be accomplished with (series inductive coupling) or without (shunt inductive coupling) galvanic isolation to the power cable [97]. In the inductive PLC coupler, electric current flows through the winding coil and yields an electromagnetic field which inductively loads the signal into the conductor, as showed in Fig. 9, where $T_{1}$ and $T_{2}$ are inductive couplers. Structurally, it consists of a magnetic core with gaps and the output coil is connected to the transceiver, both of which are wound around the core. Its installation does not require lockout of the electric power grid because there is no electric connection between power cables and the PLC coupler.

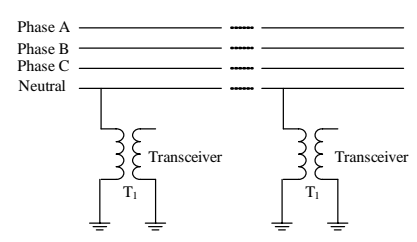

(a)

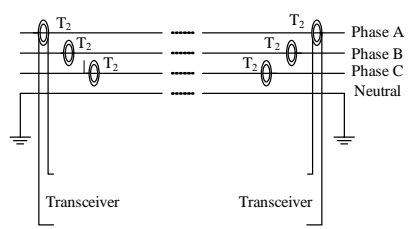

(b)
Fig. 9: (a) serial inductive coupler and (b) shunt inductive coupler.

In inductive PLC couplers, the mains current can flow unlimited through the magnetic core clamped over the wires as shown in [98]. Toroidal transformers typically have copper wire wrapped around a toroidal core so that the magnetic flux remains trapped in the coil and does not leak out. The properties of toroidal core current transformers, such as maximum transmissible primary current, amplitude, phase error as well as linearity, are basically determined by the material used in the core [99]. High magnetic coupling coefficient values are desirable in inductive couplers for offering low insertion loss and return loss, as well as for covering wide frequency bandwidth. In [100], insertion losses of inductive couplers based on Rogowski coil and a conventional (ferrite based) inductive coupler were compared. The authors measured the variation of insertion loss response of an inductive coupler in high current test in the frequency range of $1.7 \mathrm{MHz}$ and 40 $\mathrm{MHz}$. The performance of couplers based on Rogowski coil is stable because the relative permeability of an implemented coupler is very low and it is not saturated according to an increase of current.

The inductive coupling offers many degrees of freedom for system design by varying geometric parameters to tune parasitic elements, such as the crossover capacitance between the spirals, the magnetic coupling coefficient, inductance ratio and impedance terminations. The geometry and electromagnetic properties of coiled wire over the ferrite core result in a high impedance for high-frequency signals. In [101] was shown that the inductance of a choke depends not only on the geometry of the windings and the core but also on the permeability of the core material. The distributed capacitance between the winding turns acts as a shunt capacitor on the inductor resulting in the occurrence of resonance at a given frequency. Furthermore, the inductive PLC coupler shows good performance in low impedance power cable and yields low energy radiation [102]. The coupling efficiency varies according to distinct factors such as the shape and magnetic characteristics of the magnetic core, the length of the gaps and the characteristic impedance of power cable [102].

It is important to emphasize that the nonlinearity of the magnetic material together with the magneto-motive force rising from zero to a maximum, twice each cycle of the mains signal, causes distortions, such as amplitude modulation of the transmitted and received signals.

3) Resistive: The resistive coupler is constituted by a voltage divider, a bandpass filter and an amplifier. The voltage divider drops the voltage signal from the power cable and, in the following, the bandpass filter attenuates the out of band signals. Finally, the amplifier boosts the voltage signal amplitude for a further analog-to-digital conversion. Theoretically, injection and extraction of signals into/from the power cable are possible; however, only the extraction of the signal seems to be practicable. For instance, [21] proposed a circuit for a resistive coupler, see Fig. 10. As we can see, it is constituted by a voltage divider, a buffer, a band-pass filter and an amplifier. Due to its nature, the resistive PLC coupler may be suitable for coupling narrowband PLC system in low-voltage electric power grids.

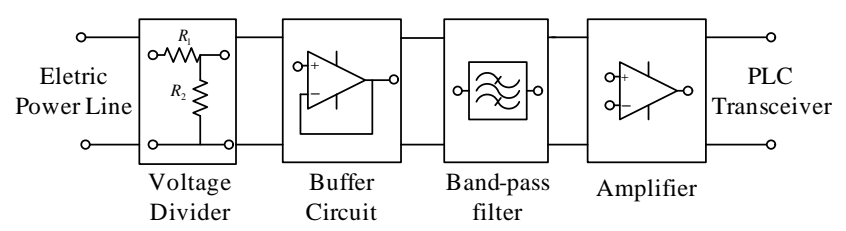

Fig. 10: The block diagram of the resistive PLC coupler.

4) Antenna: The first form of coupling PLC transceivers to power cables was based on an antenna due to its simplicity, low cost and easy installation and operation [18]. Fig. 11 shows a typical antenna coupling. These antennas were mounted below the power lines on the tower structure and the high-frequency carrier signal was induced in parallel with the power cable. The size of antennas exceeded 300 feet in length and was designed to the carrier frequency employed for voice communication [103]. However, coupling antennas are quite inefficient because there is a lot of power lost in the coupling with the electric power grid.

In 1921, AT\&T began to develop the first PLC transceiver with an antenna coupling (analog modulation, simplex transmission and transmission power of $50 \mathrm{~W}$ ) for voice signals communication, control, operation and maintenance of the distribution electric power system. However, problems such as impedance mismatching between the antenna and the power cable and the length of the antenna for working with the 
low frequencies motivated the development of capacitive and inductive couplers.

Currently, the use of antenna coupling is under investigation for broadband PLC to offer mobility to PLC users, introducing both PLC and wireless transceivers called hybrid PLC-wireless [19], [20], [104]. Furthermore, the power cable can be seen as an antenna for the propagation of RF signals, such as WiFi, [105]-[107] which can reduce the investment with cabling and repeaters. In [108], the authors characterized the LV power cable to use as an antenna to propagate RF signals in ultra-highfrequency (UHF). The range of tested frequencies in power cable extends to $550 \mathrm{MHz}$ beyond the current IEEE $802.11 \mathrm{~g}$ standard which has a center frequency of $2.45 \mathrm{GHz}$ and an $80 \mathrm{MHz}$ frequency bandwidth. It experimentally demonstrated that LV electric power grids can be used as an antenna in the UHF in the frequency range from $1 \mathrm{GHz}$ to $3 \mathrm{GHz}$.

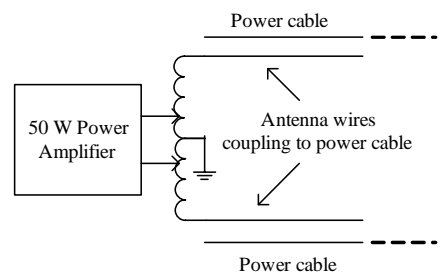

Fig. 11: Equivalent circuit of antenna wires.

\section{B. Based on voltage level}

Regardless of the electric power grids to be AC or DC, the coupling can be classified according to their voltage level as follows [109]:

1) High voltage: The coupling capacitor is the most widely used component which enables the signal coupling to and from the HV power cable [110]. Standards have been established for ratings and tests power capacitor (i.e., isolation) which must be met by coupling capacitors in HV. The requirements and essential characteristics of capacitive coupling for $\mathrm{HV}$ have been standardized in ANSI C93.1 [111].

In [112], the author describes capacitive and SISO couplers for HV transmission lines designed for narrowband and broadband PLC transceivers between the phase conductor and the earth. Basically, there are two types of phase to ground narrowband resonant schemes for coupling. Fig. 12 shows the single-frequency and the two-frequency resonant circuits that implement such scheme.

The modeling approach and analysis of a component influence on the performance of a PLC transceiver show the behavior of complex coupling with many co-existing carrier frequencies are discussed in [113]. Also, it addressed the development and application of a PLC modem components, including single and double-frequency line traps, line tuning units, coupling capacitor voltage transformers (CCVTs), transmitters, receivers, balanced and skewed hybrids and signal level probes in an $115 \mathrm{kV}$ transmission line.

The inductive coupling for $\mathrm{HV}$ transmission lines is more sensitive to HV fluctuations, impulsive noise, corona effects and voltage arcing between phases than capacitive coupling

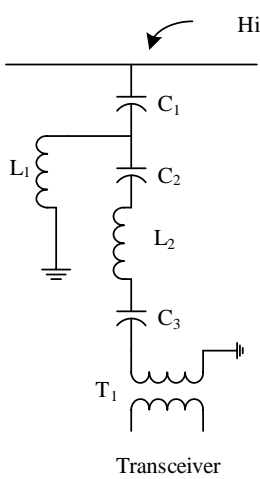

(a)

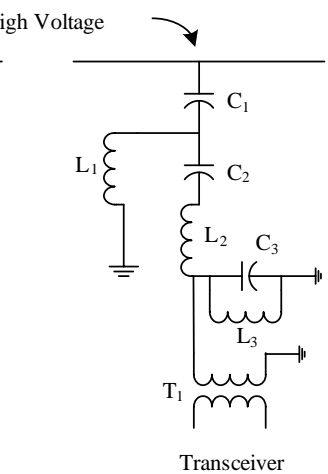

(b)
Fig. 12: Typical coupling schemes for HV transmission lines. (a) Phase-to-ground coupled single frequency narrowband and (b) Phase-to-ground coupled two frequency narrowband.

because it results in the saturation of the core [114]. Also, it provides more insertion loss than capacitive coupling. On the other hand, the greatest advantages of the inductive couplers, when compared to capacitive couplers, are: during installation, there is no interruption in the electricity supply, low cost of production and installation and the galvanic isolation among the HV transmission lines and the transceiver circuit [115]. The inductive coupling in HV transmission lines acts as an electrical protection. For instance, if there is an increase in electrical current in the HV transmission lines due to shortcircuit or short discharges, the voltage is limited by the saturation of the magnetic flux inside the core of the inductive coupler, due to the saturation of the RF transformer core.

2) Medium voltage: In recent years, the idea of using the existing MV electric distribution grids as a medium for data communications became a reality. In fact, such infrastructure can be used to constitute data communication backbone to meet protection, metering, distribution, automation and control, by connecting several substations [116].

The MV coupler must withstand high differential voltages among phases and earth. As a consequence, it requires special components, which make these devices more expensive and more complex to implement than LV couplers but much less expensive than HV couplers. The requirements for the installation of capacitors in MV must also follow the ANSI C93.1 [111].

Similar to HV PLC couplers, MV PLC couplers must be in compliance with the requirement of the utility and applicable regulation (e.g. insulation level of feeding cable, grounding, mechanical strength, lifetime and climatic category). In the case of switching the MV switchboard or in the case of short circuit, the MV PLC coupler must be able to support transient currents higher than $20 \mathrm{kA}$ with no mechanical damage or change of frequency response not exceeding $1.5 \mathrm{~dB}$ [117].

In [118], [119], a capacitive SISO PLC coupler for broadband PLC was proposed. Fig. 13 shows a MV PLC coupler device which can work with voltage levels as high as 11 $\mathrm{kV}$. In this proposal, the PLC coupler is connected to phases insulated from the power grid by line trap circuits. The line trap circuit is a parallel resonant circuit, mounted in-line on 
AC transmission power lines to prevent the transmission of high-frequency carrier signals of power line communication to unwanted destinations. It does not affect the low-frequency signal of the AC power line but offers a high impedance to the high-frequency signal of the PLC transceivers. Two RF transformers are used to achieve the desired isolation. The series combination of $L_{S}$ and $C_{S}$ form a band-pass filter to block the $\mathrm{AC}$ power line and present a low impedance for the signal of PLC transceivers. The resonant frequencies of the three circuits $\left(L_{S}, C_{S}, L\right.$ and $C$ ) are designed as the midband frequency of the PLC coupler. The MOV together with $D_{Z 1}$ and $D_{Z 2}$ (Zener diodes) are responsible for the electric protection.

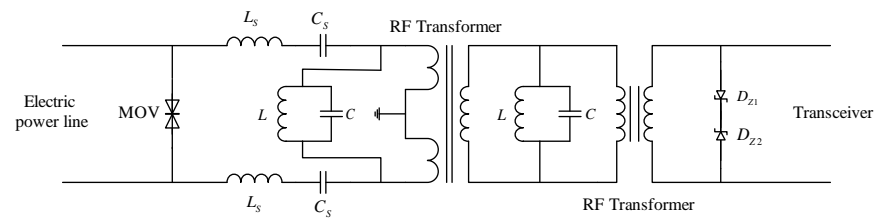

Fig. 13: Typical capacitive coupling circuit for MV.

When the inductive PLC coupler comes to matter, there are two options available for installation: the inductive PLC coupler is clamped around an MV power cable, or the inductive PLC coupler is serially connected between the neutral line of the MV circuit and the ground wire if it is available [115]. The former has the advantage of offering galvanic isolation from the power cable and its installation is done without powering off. On the other hand, its disadvantage is that high current on the MV power cable causes the magnetic saturation of the RF transformer and, as a consequence, the insertion of relevant loss. The latter has the advantage of facing low saturation magnetic flux density because of the low-level current flowing between neutral and ground lines resulting in low insertion loss. Moreover, it offers an interesting cost effective solution because the PLC coupler has a small size in comparison to the capacitive MV PLC coupler [97]. In addition, due to its characteristics, the distance for signal transmission can be long [120]-[122].

3) Low voltage: Typical LV power cables consist of three conductors, a phase, a neutral and ground. Two of these three conductors can be used to create a communication channel. The scenario for LV electric power grids is different compared with HV and MV ones, as discussed in [123] because the $\mathrm{LV}$ power cable is derived from secondary windings of the $\mathrm{MV} / \mathrm{LV}$ power transformer and the energy is delivered to a large number small consumers. The dynamic of the loads in LV electric power grids yields emissions and interference associated with appliances, motors and other loads, which make them the most hostile environment for data communication purposes [124], [125] and difficulty impedance matching among PLC transceivers and power lines. Due to the low cost of LV PLC couplers, some features can be improved, such as impedance matching with electric power grids by using, for instance, the RF transformer ratio transformation or the design of adaptive matching impedance and selective filters that is capable of attenuating unwanted frequencies which may cause interference with the PLC system.

In [70] it was shown that the analog filter is the key issue of LV PLC capacitive couplers. The authors showed how to build the filter circuit model for LV, and the Butterworth bandpass filter which frequency band is from $1 \mathrm{MHz}$ up to $30 \mathrm{MHz}$ was designed. The main issues for designing a capacitive SISO PLC coupler was discussed in Section II.

The inductive coupling has been studied in motor cable communication [126] for a voltage of $400 V_{D C}$. The inductive PLC coupler for motor cable communication is tuned for the low voltage direct current (LVDC) [127]. The inductive couplers are not commonly used in consumer applications, in which the connection with the power cable is made through an electrical wall outlet. The typical circuit of LVDC and the basic structure of the proposed PLC-base communication architecture can be accessed in [128]. Also, we point that an LVDC is very common in vehicles.

\section{Based on type of voltage}

The use of PLC in AC electric power grids is widespread, but the voltage of electric circuits can be classified into two categories: AC and DC. However, despite the popularity of PLC system over AC electrical circuits, there are a lot of researches focused on facilitating communication over DC electric circuits. In [93], the DC PLC coupler is developed on the basis of AC PLC coupler. Essentially, the fundamental structure of a DC PLC coupler is very similar to the AC PLC coupler. In [129] were investigated the possibility of using the PLC technology to transmit data along differential DC power buses employed in spacecraft.

The inductive and capacitive PLC coupler can be designed to work in different levels of voltage in AC or DC electrical circuits. A DC PLC coupler uses a series capacitor connected with the power cable such as an AC PLC coupler does. The capacitor blocks the AC or DC mains signal and passes high frequencies for a wide range of frequencies. In inductive coupling without galvanic contact with the DC bus voltage, the inductive PLC coupler, installed in parallel with DC bus, injects the PLC signal following the principle of magnetic induction, which is exploited in inductive coupling, see Section III-A-2.

The applicability of narrowband and broadband PLC for the LVDC electrical circuits communication is shown in [130]. In [131] narrowband capacitive couplers that are differentially connected between the neutral and DC conductors in bipolar $\pm 750 V_{D C}$ is proposed. An inductive coupler is used in [132] for analysis of noise in broadband PLC in DC power cables.

\section{Based on frequency band}

The PLC system has a range of frequency bands allocated to narrowband and broadband PLC data communications. Therefore, PLC couplers can be classified as narrowband (frequency range between $0 \mathrm{kHz}$ and $500 \mathrm{kHz}$ ) [38], [52] and broadband (frequency range from $1.7 \mathrm{MHz}$ to $500 \mathrm{MHz}$ ) [102], [118]. The frequency bandwidth is determined by low and high cut-off frequencies at $3 \mathrm{~dB}$ attenuation and can be modified in function of filter design. The parameters of the 
RF transformer, analog filter and electric protection must be designed in accord with the chosen frequency band desired [37], [43].

\section{E. Based on propagation mode}

Power cable wiring installations in a residence make use of three wires, namely phase, neutral and protective ground, which can be modeled as parallel conductors sheathed in a dielectric material [133]. PLC couplers can inject a signal in the power cable along the wire in two ways: DM and CM [134].

The DM is the normal current conduction mode by a twowire circuit. The current flows in a wire in phase opposition of the other wire, the electromagnetic fields generated by the currents actually cancel out each other. On the other hand, in the CM, the current goes through all the wires in the same direction causing the addition of electromagnetic fields, yielding significant levels of electromagnetic interference (EMI) [135].

Fig. 14 shows a high-frequency equivalent circuit of a PLC channel illustrating the creation of CM signals due to current $I_{c m}$ and voltage $V_{c m}$ with respect to the ground, originated unintentionally in an unbalanced electric power cable. It creates a parasitic capacitance with respect to ground and causes a CM current to return to the source through the parasitic capacitance $\left(C_{\text {para }}\right)$. In this model, we have a three-wire power line cable in which the Ground wire is the reference conductor. The $I_{d m}$ current flows in the Phase wire from/to PLC Couplers and returns via the Neutral wire. The $I_{c m}$ currents flow from/to PLC Couplers in the Live and Neutral wires and return to PLC Couplers via the Ground wire. The $I_{c m}$ flows back to the location where it was converted via unwanted parasitic components $C_{\text {para }}$, ground and any other asymmetry.

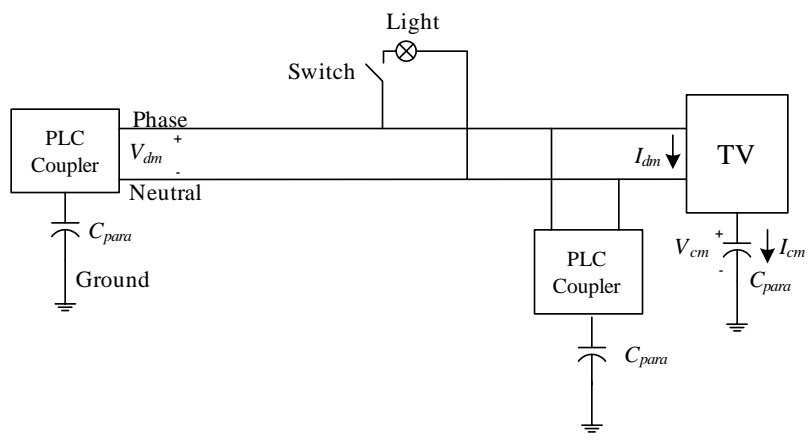

Fig. 14: High frequency equivalent circuit of a DM.

\section{F. Based on number of connections}

The wires in HV, MV and LV electric power grids can be used to provide more than one path for data communication. Hence, the coupling circuits can be classified according to the number of connections. The power cable in $\mathrm{HV}$ and MV consists of three phase wires where the same signal can be sent by three different paths. The power cable in $\mathrm{LV}$ is composed of three phase conductors and one neutral conductor and, therefore, there are four paths available for data
TABLE I: Types of connections.

\begin{tabular}{|c|c|c|}
\hline Mode & Transformer $($ Tx $)$ & Transformer $(R x)$ \\
\hline 1X1 (SISO) & $P-N$ & $P$ \\
\hline 1X2 (SIMO) & $P-N$ & $P, C M$ \\
\hline 1X3 (SIMO) & $P-N$ & $P, N, P E$ \\
\hline 1X4 (SIMO) & $P-N$ & $P, N, P E, C M$ \\
\hline 2X2 (MIMO) & $P-N, P-P E$ & $P, P E$ \\
\hline 2X3 (MIMO) & $P-N, P-P E$ & $P, N, P E$ \\
\hline 2X4 (MIMO & $P-N, P-P E$ & $P, N, P E, C M$ \\
\hline
\end{tabular}

communication [136], [137]. In this context, the connection between two PLC transceivers can be classified in terms of SISO, single input multiple outputs (SIMO), multiple inputs multiple outputs (MISO) and multiple inputs multiple outputs (MIMO).

The SISO coupling is the simplest form of coupling with power cable. In LV, the connection of PLC couplers can be performed by the phase and neutral wires or phase to phase wires. Such connection in HV and MV occurs among phases. The advantage of the SISO PLC coupler is its simplicity and low cost. The majority of PLC systems are SISO.

To have SIMO and MISO PLC couplers, connecting at HV and MV, all three phases wires must be available. The SIMO PLC coupler is connected in two phases to transmit the signal from the transceiver circuit and it receives the signal in three phases. In that case, the signal is induced in the third phase.

In MIMO coupling at $\mathrm{HV}$ and $\mathrm{MV}$, all three phase wires can be used, simultaneously. The MIMO PLC coupler is connected in three phases to transmit the signal and it receives the signal in two phases. For LV electric power grids the electrical protective wire and protective earth (PE) allows the creation of another path between two PLC couplers [138]. Table I and Fig. 15 show the configuration mode of SISO, SIMO and MIMO. In [138], the authors show the results of channel measurements motivated to implement a MIMO PLC equivalent circuit explaining the creation and reception of $\mathrm{CM}$ signals. Two RF transformers are connected in triangle and star configuration providing several paths for data communication.

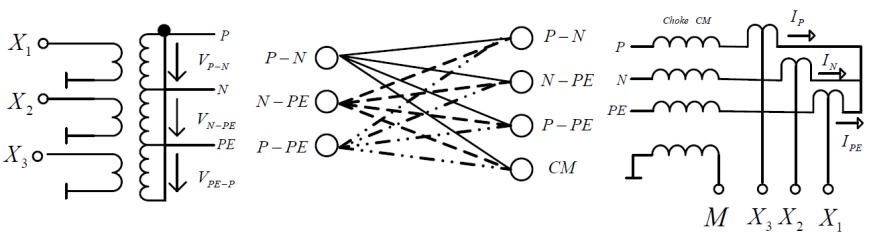

Fig. 15: MIMO PLC Coupler.

\section{FUTURE WORKS}

Although there is a scarce effort toward PLC coupling research, the coupling unit is perhaps one of the most important components in a PLC transceiver. Therefore, the design of a PLC coupler capable of handling the complexity, dynamic and diversity of electric power system is a challenging issue for the PLC research community. With this regards, in the following, we enumerate some important question deserving more attention in order to come up with improved coupling solution: 
- The measurement of access impedances is of particular importance since it is necessary for designing efficient and effective couplers, for improving the performance of the PLC coupling [129]. It is important to know time and frequency behaviors of resistive and reactive components of such impedance. Lack of this very important information has led to the design of poor quality and low efficient coupling devices in the past.

- Research and development of new components and material for electrical protection of transceiver circuits for broadband PLC. Very limited research efforts have been expended in the area of special materials for couplers, especially when compared to the work on materials for the design of advanced transformers. For instance, the use of nanocrystalline magnetic materials to substitute the ferrite and nanomaterials for capacitive devices. This kind of material will considerably reduce the cost of current coupling devices used in medium and high voltage electric power systems.

- The design of low-cost surge protective components when the frequency is over $35 \mathrm{MHz}$. Our review showed that GDT and TVSP are the most recommended if the mains voltage is high; however, their cost may limit the use of them in commercial coupling devices.

- The development of simple and effective coupling circuits that is capable of performing impedance matching to maximize power transfer. The introduction of such kind of coupler will result in a remarkable improvement of PLC technology.

\section{CONCLUSION}

A review on the use of couplers to enable data communications over AC and DC electric power grids have been presented. PLC couplers enable data communications over power lines by injecting/extracting data signals into or from these power lines either by differential mode or by common mode. The review showed that the introduction of couplers to connect PLC transceivers to electric power circuits could be used to minimize both signal distortion and insertion loss to improve PLC systems performance.

In this regard, we isolated the key issues related to the design of a PLC coupler, such as the PCB design, insertion and return loss, galvanic isolation, impedance matching, filtering and protection. Additionally, we provided a classification of PLC couplers based on the type of physical coupling, voltage levels, frequency bandwidth, propagation mode and number of connections. These fundamental concepts are provided to suffice basic understanding about some relevant issues related to the design of PLC couplers.

Finally, we pointed out some important research questions whose investigations can result in the design of a new generations of efficient and effective PLC couplers.

\section{ACKNOWLEDGMENT}

The authors would like to thank CNPq, CAPES, FAPEMIG and Smarti9 for providing technical and financial support.

\section{REFERENCES}

[1] M. Schwartz, "Carrier-wave telephony over power lines: Early history [history of communications]," IEEE Communications Magazine, vol. 47, no. 1, pp. 14-18, Jan. 2009, doi: 10.1109/MCOM.2009.4752669.

[2] H. C. Ferreira, L. Lampe, J. Newbury, and T. G. Swart, Power Line Communications Theory and Applications for Narrowband and Broadband Communications over Power Lines. New York: John Wiley and Sons, 2010.

[3] H. Meng, S. Chen, Y. Guan, C. Law, P. So, E. Gunawan, and T. Lie, "Modeling of transfer characteristics for the broadband power line communication channel," IEEE Trans. on Power Delivery., vol. 19, no. 3, pp. 1057-1064, Jul. 2004, doi: 10.1109/TPWRD.2004.824430.

[4] A. Pinomaa, J. Ahola, A. Kosonen, and P. Nuutinen, "Power line communication network for a customer-end AC grid in an LVDC distribution system," in Proc. IEEE International Conference on Smart Grid Communications, Nov. 2014, pp. 224-229, doi: 10.1109/SmartGridComm.2014.7 007650.

[5] S. Chen, M. Setta, X. Chen, and C. G. Parini, "Ultra wideband powerline communication (PLC) above $30 \mathrm{MHz}$," Institution of Engineering and Technology Communications, vol. 3, no. 10, pp. 1587-1596, Oct. 2009, doi: 10.1049/iet-com.2008.0557.

[6] E. A. Teixeira, E. L. Rege, F. S. Marques, E. M. de Souza, T. M. Johnson, and M. V. Ribeiro, "Regulation issues relating to broadband PLC: A Brazilian experience and perspective," in Proc. IEEE International Symposium on Power Line Communications and Its Applications, Mar. 2007, pp. 523-527, doi: 10.1109/ISPLC.2007.371 179.

[7] K. Dostert, Powerline Communications. Prentice Hall, 2001.

[8] J. Granado, A. Torralba, and J. Chavez, "Using broadband power line communications in non-conventional applications," IEEE Trans. on Consumer Electronics, vol. 57, no. 3, pp. 1092-1098, Aug. 2011, doi: 10.1109/TCE.2011.6018860.

[9] H. Ferreira, H. Grove, O. Hooijen, and A. J. Han Vinck, "Power line communications: an overview," in Proc. IEEE African Electrical Technology Conference, vol. 2, Sep. 1996, pp. 558-563, doi: 10.1109/AFRCON.1996.562949.

[10] X. Carcelle, Power Line Communications in Practice. New York: Artech House, 2006.

[11] T. R. Oliveira, W. A. Finamore, and M. V. Ribeiro, "A sounding method based on OFDM modulation for PLC channel measurement," in Proc. IEEE International Symposium on Power Line Communications and Its Applications, Mar. 2013, pp. 185-190, doi: 10.1109/ISPLC.2013.6525 847.

[12] A. A. M. Picorone, T. R. Oliveira, and M. V. Ribeiro, "PLC channel estimation based on pilots signal for OFDM modulation: A review," IEEE Latin America Transactions, vol. 12, no. 4, pp. 580-589, Jun. 2014.

[13] J. Dias, F. P. V. de Campos, M. L. R. de Campos, E. L. Pinto, and M. V. Ribeiro, "Time-varying channel characterization based on SVM under the impulsive noise presence," in Proc. IEEE International Symposium on Power Line Communications and Its Applications, Mar. 2010, pp. 261-266, doi: 10.1109/ISPLC.2010.5 479885.

[14] G. R. Colen, H. Schettino, D. Fernandes, L. M. Sirimarco, F. P. Campos, W. A. Finamore, H. A. Latchman, and M. V. Ribeiro, "A temporal compressive resource allocation technique for complexity reduction in plc transceivers," Trans. on Emerging Telecommunications Technologies, vol. 1, no. 1, pp. 1-12, May 2015, doi: 10.1002/ett.

[15] M. V. Ribeiro, G. R. Colen, Z. Quan, H. V. Poor et al., "Clusteredorthogonal frequency division multiplexing for power line communication: when is it beneficial?" IET Communications, vol. 8, no. 13, pp. 2336-2347, Apr. 2014, doi:10.1049/iet-com.2014.0056.

[16] M. V. Ribeiro, R. R. Lopes, J. M. T. Romano, and C. A. Duque, "Impulse noise mitigation based on computational intelligence for improved bit rate in PLC-DMT," IEEE Trans. on Power Delivery, vol. 21, no. 1, pp. 94-101, Jan. 2006, doi: 10.1109/TPWRD.2005.852271.

[17] R. M. Oliveira, M. S. Facina, M. V. Ribeiro, and A. B. Vieira, "Performance evaluation of in-home broadband plc systems using a cooperative MAC protocol," Computer Networks, vol. 95, pp. 62-76, Dec. 2016, doi: 10.1016/j.comnet.2015.12.004.

[18] C. A. Boddie, "Telephone communication over power lines by high frequency currents," Institute of Radio Engineers, vol. 15, no. 7, pp. 559-640, Jul. 1927, doi: 10.1109/JRPROC.1927.221237.

[19] T. R. Oliveira, C. A. G. Marques, M. S. Pereira, S. L. Netto, and M. V. Ribeiro, "The characterization of hybrid PLC-wireless channels: A preliminary analysis," in Proc. IEEE International Symposium on 
Power Line Communications and Its Applications, Mar. 2013, pp. 98102, doi: 10.1109/ISPLC.2013.6525832.

[20] T. R. Oliveira, F. J. A. Andrade, A. A. M. Picorone, H. A. Latchman, S. L. Netto, and M. V. Ribeiro, "Characterization of hybrid communication channel in indoor scenario," Journal of Communication and Information System., vol. 31, no. 1, pp. 224 - 235, Nov. 2016.

[21] Z. W. Swana, P. A. J. van Rensburg, and H. C. Ferreira, "Is resistive coupling feasible for the reception of power-line communications data?" in Proc. International Symposium on Power Line Communications and Its Application, May 2015, pp. 47-52.

[22] J. Anatory and T. N., Broadband Power-line Communication Systems Theory and Applications. Boston: Wit Press, 2010.

[23] L. Simonovich, "Relative permittivity variation surrounding PCB via hole structures," in Proc. IEEE Workshop on Signal Propagation on Interconnects, May 2008, pp. 1-4, doi: 10.1109/SPI.2008.4558 362.

[24] D. Moongilan, "Minimizing radiated emissions from PCBs using gridlike ground plane impedance matching techniques," in Proc. International Symposium on Electromagnetic Compatibility, vol. 3, Aug. 2005, pp. 971-976, doi: 10.1109/ISEMC.2005.1513666.

[25] G. Smithson, "Practical RF printed circuit board design," in Proc. How to Design RF Circuits, IEE Training Course, Jul. 2000, pp. 11/1-11/6, doi: 10.1049/ic:20 000150

[26] T. T. Yan and M. Z. M. Jenu, "Capacitive and inductive couplings of PCB traces," in Proc. Telecommunication systems Congresses, vol. 1, Sep. 2000, pp. 186-191, doi: 10.1109/TENCON.2000.893567.

[27] M. Rajesh, P. Bhartia, and I. J. Bahl, RF and Microwave Coupled Line Circuits, 2nd, Ed. Boston: Artech House, 2007.

[28] M. I. Montrose, Eletromagnetic Compliance and the Printed Circuit Board: Design, Theory and Layout Made Simple. New York: Wiley Interscience, 1999.

[29] R. Mongia, I. Bahl, and P. Jit Bharitia, RF and Microwave Coupled-line Circuits. Norwood: Artech House,, 1999.

[30] S. C. Thierauf, High-Speed Circuit Board Signal Integrity. New York: Artech House, 2004

[31] G. Gonzalez, R. Linares Y.M., J. De La Rosa, and W. Fonseca, "Ground plane impedance analysis of printed circuit board," in Proc. IEEE International Symposium on Electromagnetic Compatibility, vol. 2, Aug. 1999, pp. 712-715, doi: 10.1109/ISEMC.1999.810 105.

[32] D. Brooks, Signal Integrity Issues and Printed Circuit Board Design. Boston: Prentice Hall, 2003

[33] R. Ludwig and G. Bogdanov, RF Circuit Design, Theory and Applications. New Delhi, India: Prentice Hall, 2009.

[34] K. Fezzani, C. Rebai, and A. Ghazel, "Analysis and optimization of power line coupling circuit for CENELEC-PLC modem," in Proc. IEEE International Conference on Electronics, Circuits and Systems, Dec. 2006, pp. 676-679, doi: 10.1109/ICECS.2006.379 879.

[35] Z. Tao, Y. Xiaoxian, and Z. BaoHui, "Influence and modification of coupling device on low voltage power network channel characterization measurement," in Proc. IEEE Power and Energy Society Transmission and Distribution Conference and Exhibition: Asia and Pacific,, Apr. 2005, pp. 1-5, doi: 10.1109/TDC.2005.1547 109.

[36] W. A. Davis and K. Agarwal, Radio Frequency Circuit Design, K. Chang, Ed. New York: John Wiley and Sons, 2001.

[37] P. J. Van Rensburg and H. C. Ferreira, "Coupling circuitry: understanding the functions of different components," in Proc. International Symposium in Power Line Communications and Its Applications, vol. 1, Mar. 2003, pp. 204-209.

[38] P. Van Rensburg and H. Ferreira, "Coupler winding ratio selection for effective narrowband power-line communications," IEEE Trans. on Power Delivery, vol. 23, no. 1, pp. 140-149, Jan. 2008, doi: 10.1109/TPWRD.2007.905790

[39] H. Ferreira and P. Van Rensburg, "Dual coupler for dedicated switching with loads," in Proc. IEEE International Symposium on Power Line Communications and Its Applications, Mar. 2007, pp. 53-58, doi: 10.1109/ISPLC.2007.371 097.

[40] A. C. M. De Queiroz, "Band-pass multiple resonance networks," in Proc. Midwest Symposium on Circuits and Systems, vol. 3, Jul. 2004, pp. 275-278, doi: 10.1109/MWSCAS.2004.1354347.

[41] P. Van Rensburg and H. Ferreira, "Design of a bidirectional impedanceadapting transformer coupling circuit for low-voltage power-line communications," IEEE Trans. on Power Delivery, vol. 20, no. 1, pp. 64-70, Jan. 2005, doi: 10.1109/TPWRD.2004.835260(410) 2.

[42] M. K. Kazimierczuk, Radio Frequency Circuit Designg. New York: John Wiley and Sons, 2009.

[43] V. Dumbrava and L. Svilainis, "RF transformer model parameters measurement," in Proc. Design and Diagnostics of Electronic Circuits and Systems, Apr. 2007, pp. 1-5, doi: 10.1109/DDECS.2007.4 295298.
[44] M. S. Damnjanovic, L. D. Zivanov, S. M. Djuric, A. M. Maric, A. B. Menicanin, G. J. Radosavljevic, and N. V. Blaz, "Characterization and modelling of miniature ferrite transformer for high frequency applications," Microelectronics International Magazine, vol. 29, no. 2, pp. 83-89, Jan. 2012.

[45] J. Biernacki and D. Czarkowski, "High frequency transformer modeling," in Proc. IEEE International Symposium on Circuits and Systems, vol. 2, May 2001, pp. 676-679, doi: 10.1109/ISCAS.2001.921 401.

[46] Y. Sun and G. Amaratunga, "High-current adaptive impedance matching in narrowband power-line communication systems," in Proc. IEEE International Symposium on Power Line Communications and Its Applications, Apr. 2011, pp. 329-334, doi: 10.1109/ISPLC.2011.5 764417

[47] Y. Xiaoxian, Z. Tao, Z. BaoHui, Y. Fengchun, D. Jiandong, and S. Minghui, "Research of impedance characteristics for mediumvoltage power networks," IEEE Trans. on Power Delivery, vol. 22, no. 2, pp. 870-878, Apr. 2007, doi: 10.1109/TPWRD.2006.881573.

[48] F. Corripio, J. Arrabal, L. del Rio, and J. Munoz, "Analysis of the cyclic short-term variation of indoor power line channels," IEEE Journal on Selected Areas in Communications, vol. 24, no. 7, pp. 1327-1338, Jul. 2006, doi: 10.1109/JSAC.2006.874402.

[49] A. Tonello, F. Versolatto, and A. Pittolo, "In-home power line communication channel: statistical characterization," IEEE Trans. on Communications, vol. 62, no. 6, pp. 2096-2106, Jun. 2014, doi: 10.1109/TCOMM.2014.2317790.

[50] L. G. S. Costa, A. A. M. Picorone, A. C. M. de Queiroz, V. L. R. Costa, and M. V. Ribeiro, "Caracterização da impedância de acesso à rede de energia elétrica residencial para uso em sistemas PLC," XXXIII Simpósio Brasileiro de Telecomunicações, pp. 1-5, Sep. 2015.

[51] G. R. Colen, C. A. G. Marques, T. R. Oliveira, F. P. V. de Campos, and M. V. Ribeiro, "Measurement setup for characterizing low-voltage and outdoor electric distribution grids for PLC systems," in Proc. Conference On Innovative Smart Grid Technologies Latin America, Apr. 2013, pp. 1-5, doi: 10.1109/ISGT-LA.2013.6554476.

[52] H. Gassara, M. Bali, F. Duval, F. Rouissi, and A. Ghazel, "Coupling interface circuit design for experimental characterization of the narrowband power line communication channel," in Proc. IEEE International Symposium on Electromagnetic Compatibility, Aug. 2012, pp. 1-6, doi: 10.1109/ISEMC.2012.6351760.

[53] R. Vines, H. Trussell, K. Shuey, and J. O'Neal, J.B., "Impedance of the residential power-distribution circuit," IEEE Trans. on Electromagnetic Compatibility, vol. EMC-27, no. 1, pp. 6-12, Feb. 1985, doi: 10.1109/TEMC.1985.304239.

[54] E. Karadeniz and I. Cavdar, "Measurements of impedance and attenuation at CENELEC bands for power line communications systems," Sensors, pp. 8027-8036, Dec. 2008.

[55] J. Malack and J. Engstrom, "RF impedance of United States and European power lines," IEEE Trans. on Electromagnetic Compatibility, vol. EMC-18, no. 1, pp. 36-38, Feb. 1976, doi: 10.1109/TEMC.1976.303453

[56] J. R. Nicholson and J. Malack, "RF impedance of power lines and line impedance stabilization networks in conducted interference measurements," IEEE Trans. on Electromagnetic Compatibility, vol. EMC-15, no. 2, pp. 84-86, May 1973, doi: 10.1109/TEMC.1973.303254.

[57] H. Tian, R. Yuan, F. Li, Z. Huang, S. Wang, S. Li, and K. Zhong, "Measurement on narrow band power line communication channel impedance of distribution network," in Proc. International Conference on Consumer Electronics, Communications and Networks, Apr. 2011, pp. 454-457, doi: 10.1109/CECNET.2011.5 768876.

[58] R. Araneo, S. Celozzi, and G. Lovat, "Design of impedance matching couplers for power line communications," in Proc. IEEE International Symposium in Electromagnetic Compatibility, Aug. 2009, pp. 64-69, doi: 10.1109/ISEMC. 2009.5284630.

[59] L. Qi, S. Jingzhao, and F. Zhenghe, "Adaptive impedance matching in power line communication," in Proc. International Conference in Microwave and Millimeter Wave Technology, Aug. 2004, pp. 887-890, doi: 10.1109/ICMMT.2004.1411673.

[60] S. Despande, I. Prasanna, and S. Panda, "An adaptive impedance matching technique for narrowband power line communication in residential smart grids," International Journal of Engineering Research and Technology, vol. 2, p. 9, Sep. 2013.

[61] A. A. Atayero, A. A. Alatishe, and Y. A. Ivanov, "Power line communication technologies:modeling and simulation of PRIME physical layer," in Proc. World Congress on Engineering and Computer Science, vol. 2, Oct. 2012, pp. 24-26.

[62] C. P. Rui, N. N. Barsoum, A. W. K. Ming, and W. K. Ing, "Adaptive impedance matching network with digital capacitor in 
narrowband power line communication," in Proc. IEEE International Symposium on Industrial Electronics, May 2013, pp. 1-5, doi: 10.1109/ISIE.2013.6563591.

[63] C.-Y. Park, K.-H. Jung, and W.-H. Choi, "Coupling circuitary for impedance adaptation in power line communications using VCGIC," in Proc. IEEE International Symposium on Power Line Communications and Its Applications, pp. 293-298, Apr. 2008, doi:10.1109/ISPLC.2008.4510442.

[64] W.-H. Choi and C. Y. Park, "A simple line coupler with adaptive impedance matching for power line communication," in Proc. IEEE International Symposium on Power Line Communications and Its Applications, Mar. 2007, pp. 187-191, doi: 10.1109/ISPLC.2007.371 121.

[65] M. Sibanda, P. Van Rensburg, and H. Ferreira, "Impedance matching with low-cost, passive components for narrowband PLC," in Proc. IEEE International Symposium on Power Line Communications and Its Applications, Apr. 2011, pp. 335-340, doi: 10.1109/ISPLC.2011.5 764418 .

[66] M. Sibanda, P. van Rensburg, and H. Ferreira, "A compact economical plc band-pass coupler with impedance matching," in Proc. IEEE International Symposium on Power Line Communications and Its Applications, Mar. 2013, pp. 339-344, doi: 10.1109/ISPLC.2013.6525874.

[67] S. Yang, H. Li, M. Goldberg, X. Carcelle, F. Onado, and S. Rowland, "Broadband impedance matching circuit design using numerical optimisation techniques and field measurements," in Proc. IEEE International Symposium on Power Line Communications and Its Applications, Mar. 2007, pp. 425-430, doi: 10.1109/ISPLC.2007.371 162.

[68] R. Araneo, S. Celozzi, G. Lovat, and F. Maradei, "Multi-port impedance matching technique for power line communications," in Proc. IEEE International Symposium on Power Line Communications and Its Applications, Apr. 2011, pp. 96-101, doi: 10.1109/ISPLC.2011.5764 458

[69] J. Kennedy and R. Eberhart, "Particle swarm optimization," in Proc. Conference on Neural Networks, vol. 4, Nov. 1995, pp. 1942-1948, doi: 10.1109/ICNN.1995.488968.

[70] S. Wei, W. Gao, L. Zhang, and Z. Cao, "High frequency and broadband coupling characteristics of filter circuit based on low voltage power lines," in Proc. Advanced in Control Engineering and Information Science, vol. 15, pp. 1978-1982, Aug. 2011, doi: 10.1016/j.proeng.2011.08.369.

[71] H. Ueda and T. Tsuboi, "A sampling theorem for periodic functions with no minus frequency component and its application," in Proc. AsiaPacific Conference on Communications, Aug 2013, pp. 225-230, doi: 10.1109/APCC.2013.6 765946.

[72] C. Bowick, RF Circuit Design, 2nd, Ed. Burlington: Howard W. Sams and $\operatorname{Cos}, 2008$.

[73] J. Zdunek, "Generation of filter functions from a given model," Institute of Electrical Engineers, vol. 110, no. 2, pp. 282-294, Feb. 1963, doi: 10.1049/piee.1963.0041.

[74] C. P. Serra, Teoria e Projeto de Filtros. Campinas, Brazil: Cartigraf, 1983.

[75] A. C. M. de Queiroz and L. P. Caloba, "Passive symmetrical RLC filters suitable for active simulation," in Proc. IEEE International Symposium in Circuits and Systems, vol. 3, Jun. 1988, pp. 2411-2414, doi: 10.1109/ISCAS.1988.15 429.

[76] B. Zarikoff and D. Malone, "Experiments with radiated interference from in-home power line communication networks," in Proc. IEEE International Conference on Communications, Jun. 2012, pp. 34143418, doi: 10.1109/ICC.2012.6363802.

[77] E. A. Teixeira, F. S. Marques, S. G. de Araujo, E. M. de Souza, T. M. Johnson, and M. V. Ribeiro, "Modeling and performance analysis of PLC channels with external interference in outdoor and indoor environments," in Proc. IEEE International Symposium on Power Line Communications and Its Applications, Apr. 2008, pp. 222-227, doi: 10.1109/ISPLC.2008.4510428.

[78] F. Leccese, "Study and characterization of a new protection system against surges and over voltages for domestic telecommunication networks," in Proc. International Telecommunications Energy Conference, Sep. 2007, pp. 363-368, doi: 10.1109/INTLEC.2007.4 448799.

[79] V. Kamat, "Protection mechanism for modern telecommunications," in Proc. International Conference in Electromagnetic Interference and Compatibility, Dec. 1997, pp. 45-50, doi: 10.1109/ICEMIC.1997.669 756.

[80] T. Imamura and A. Ametani, "Investigation of transient induced voltage to a communication line from an overhead power transmission line," Generation, Transmission and Distribution, vol. 137, no. 2, pp. 129138, Mar. 1990.
[81] A. Gayen, A. Dhar, B. Das, and D. Poddar, "Study of the induced voltage on an overhead line due to lightning discharge," in Proc. International Conference in Electromagnetic Interference and Compatibility, Feb. 2006, pp. 353-357.

[82] W. Vijayananda and S. Alahakoon, "Surge arrester with improved reliability and protection level for low bandwidth data communication in high voltage installations," in Proc. Australasian Universities Power Engineering Conference, Dec. 2010, pp. 1-6.

[83] H. Iwao, H. Kijima, and K. Takato, "An influence on transmission characteristics of power line communication when using surge protective devices," in Proc. IEEE International Symposium in Power Line Communications and Its Applications, Apr. 2008, pp. 218-221, doi: 10.1109/ISPLC.2008.4510 427

[84] M. Hove, T. O. Sanya, A. J. Snyders, I. R. Jandrell, and H. C. Ferreira, "The effect of type of transient voltage suppressor on the signal response of a coupling circuit for power line communications," in Proc. IEEE African Electrical Technology Conference, Sep. 2011, pp. 1-6, doi: 10.1109/AFRCON.2011.6072050.

[85] M. Drabkin, "Surge protection of low-voltage AC power by MOVbased SPDs," in Proc. 10th International Conference in Harmonics and Quality of Power, vol. 1, Oct. 2002, pp. 13-16, doi: 10.1109/ICHQP.2002.1221397.

[86] J. Wei and D. Gerling, "Simulation and modelling of power passive components for EMI prediction," in Proc. IET International Conference on Power Electronics, Machines and Drives, Mar. 2012, pp. 1-6, doi: 10.1049/cp.2012.0209

[87] S. Chickamenahalli, H. Braunisch, S. Srinivasan, J. He, U. Shrivastava, and B. Sankman, "RF packaging and passives: design, fabrication, measurement, and validation of package embedded inductors," IEEE Trans. on Advanced Packaging, vol. 28, no. 4, pp. 665-673, Nov. 2005, doi: 10.1109/TADVP.2005.850510.

[88] M. Bartoli, A. Reatti, and M. Kazimierczuk, "High-frequency models of ferrite core inductors," in Proc. International Conference on Industrial Electronics, Control and Instrumentation, vol. 3, Sep. 1994, pp. 1670-1675, doi: 10.1109/IECON.1994.398065.

[89] R. Lafferty, "Measuring the self-resonant frequency of capacitors," IEEE Trans. on Components, Hybrids, and Manufacturing Technology, vol. 5, no. 4, pp. 528-530, Dec. 1982, doi: 10.1109/TCHMT.1982.1135992.

[90] H. Hrasnica, A. Haidine, and R. Lehnert, Broadband Powerline Communications: Network Design. Chinchester, England: John Wiley and Sons, 2004.

[91] I. Whyte, "Distribution network powerline carrier communication system," U.S. Patent 3942 170, Mar. 2, 1976. [Online]. Available: https://www.google.t1/patents/US3942170

[92] L. G. S. Costa, A. A. M. Picorone, A. C. M. de Queiroz, V. L. R. Costa, and M. V. Ribeiro, "Projeto e caracterização de acopladores para power line communications," in Proc. XXXIII Simpósio Brasileiro de Telecomunicações, Sep. 2015, pp. 1-5.

[93] E. Wade and H. Asada, "Design of a broadcasting modem for a dc plc scheme," IEEE/ASME Trans. on Mechatronics, vol. 11, no. 5, pp. 533-540, Oct. 2006, doi: 10.1109/TMECH.2006.882983.

[94] F. Grassi and S. Pignari, "Coupling/decoupling circuits for powerline communications in differential DC power buses," in Proc. IEEE International Symposium on Power Line Communications and Its Applications, Mar. 2012, pp. 392-397, doi: 10.1109/ISPLC.2012.6201323.

[95] M. Lienard, M. Carrion, V. Degardin, and P. Degauque, "Modeling and analysis of in-vehicle power line communication channels," IEEE Trans. on Vehicular Technology, vol. 57, no. 2, pp. 670-679, Mar. 2008, doi: 10.1109/TVT.2007.906372.

[96] A. Pinomaa, J. Ahola, A. Kosonen, and P. Nuutinen, "Homeplug green PHY for the LVDC PLC concept: Applicability study," in Proc. IEEE International Symposium on Power Line Communications and its Applications, Mar. 2015, pp. 205-210, doi: 10.1109/ISPLC.2015.7 147615.

[97] J.-J. Lee, D.-S. In, H.-M. Oh, S. Shon, and D.-H. Nam, "Neutral inductive coupling for improved underground medium voltage BPLC," in Proc. IEEE International Symposium on Power Line Communications and Its Applications, Mar. 2010, pp. 67-71, doi: 10.1109/ISPLC.2010.5 479947.

[98] J. Binkofski, "Influence of the properties of magnetic materials," in Proc. IEEE International Symposium on Power Line Communications and Its Applications, Apr. 2005, pp. 281-284, doi: 10.1109/ISPLC.2005.1 430515.

[99] C. Kikkert, "Power transformer modelling and MV PLC coupling networks," in Proc. IEEE Power and Energy Society Innovative Smart Grid Technologies Asia, Nov. 2011, pp. 1-6, doi: 10.1109/ISGTAsia.2011.6 167093. 
[100] D.-S. In, S. Shon, and J. J. Lee, "A study on the implementation of inductive coupler with Rogowski coil for BPLC," in Proc. International Conference on Advanced Technologies for Communications, Oct. 2008, pp. 327-330, doi: 10.1109/ATC.2008.4 760588.

[101] Q. Yu, T. Holmes, and K. Naishadham, "RF equivalent circuit modeling of ferrite-core inductors and characterization of core materials," IEEE Trans. on Electromagnetic Compatibility, vol. 44, no. 1, pp. 258-262, Feb. 2002, doi; 10.1109/15.990733.

[102] O. Bilal, E. Liu, Y. Gao, and T. O. Korhonen, "Design of bidirectional coupling circuit for broadband power-line communications," Journal of Electromagnetic Analysis and Applications, vol. 4, no. 4, pp. 162-166, Apr. 2012, doi: 10.4236/jemaa.2012.44021.

[103] H.-K. Podszeck, Carrier Communication over Power Lines. New York: Springer, 1972.

[104] T. R. Oliveira, "The characterization of hybrid PLC-wireless and PLC channels in the frequency band between 1.7 and $100 \mathrm{MHz}$ for data communication," Ph.D. dissertation, Federal University of Juiz de Fora, 2015.

[105] S. Jordaan, P. A. Janse van Rensburg, A. S. De Beer, and H. C. Ferreira, "Long-wire half wave dipole antenna integrated into power cabling," Australian Patent 2013101645,2 17, 2013.

[106] A. Emleh, A. de Beer, H. Ferreira, and A. J. Han Vinck, "Interference detection on powerline communications channel when in-building wiring system acts as an antenna," in Proc. International Symposium Electronic in Marine, Sep. 2013, pp. 141-144.

[107] T. R. Oliveira, F. J. Andrade, L. G. S. Costa, M. S. Pereira, and M. V. Ribeiro, "Measurement of hybrid PLC-wireless channels for indoor and broadband data communication," in Proc. XXXI Simpósio Brasileiro de Telecomunicações, vol. 1, no. 1, Sep. 2013, pp. 1-5.

[108] S. Jordaan, P. Janse van Rensburg, A. de Beer, H. Ferreira, and A. J. Han Vinck, "A preliminary investigation of the UHF properties of LV cable for WiFi over power line communications," in Proc. IEEE International Symposium on Power Line Communications and its Applications, Mar. 2015, pp. 35-40, doi: 10.1109/ISPLC.2015.7 147586.

[109] L. Lampe, T. A. M., and T. G. Swart, Power Line Communications: Principles, Standards and Applications from Multimedia to Smart Grid. New Deli: John Wiley \& Sons, 2016.

[110] "IEEE guide for power-line carrier applications," IEEE Std 6432004, Revision of IEEE Std 643-1980, pp. 1-134, Aug. 2005, doi: 10.1109/IEEESTD.2005.96284.

[111] "American national standard requirements for power-line carrier coupling capacitors and coupling capacitor voltage transformers," ANSI C93.1-1999, pp. 41-42, May 1999, doi:10.1109/IEEESTD.1999.4303014.

[112] G. Franklin, "A practical guide to harmonic frequency interference affecting high-voltage power-line carrier coupling systems," IEEE Trans. on Power Delivery, vol. 24, no. 2, pp. 630-641, Apr. 2009, doi: 10.1109/TPWRD.2008.2002700.

[113] B. A. Mork, D. Ishchenko, X. Wang, A. Yerrabelli, R. Quest, and C. Kinne, "Power line carrier communications system modeling," in Proc. International Conference on Power Systems Transients, Jun. 2005, pp. 1-6.

[114] R. Pighi and R. Raheli, "On multicarrier signal transmission for highvoltage power lines," in Proc. IEEE International Symposium on Power Line Communications and Its Applications, Apr. 2005, pp. 32-36, doi: 10.1109/ISPLC.2005.1430 460.

[115] P. Wouters and P. van der Wielen, "Effect of cable load impedance on coupling schemes for MV power line communication," in Proc. IEEE Bologna Power Technology Conference, vol. 2, Jun. 2003, pp. 7, doi: 10.1109/PTC.2003.1304318

[116] F. Campagna, M. Quarantelli, and R. Pighi, "High-frequency characterization of a medium voltage PLC transmission system," in Proc. Third Workshop on Power Line Communications, Oct. 2009, pp. 1-4.

[117] F. Issa, O. Devaux, E. Marthe, and F. Rachidi, "Influence of power switching on power line communications in medium voltage networks," in Proc. IEEE International Conference on Communications, vol. 1, Jun. 2004, pp. 114-117, doi: 10.1109/ICC.2004.1312463.

[118] S. S. Ali, A. Bhattacharya, and D. R. Poddar, "Design of bidirectional coupling circuit for broadband power-line communications," Journal of Electromagnetic Analysis and Applications, vol. 4, pp. 162-166, Apr. 2012, doi: 10.4236/jemaa.2012.44021.

[119] M. Rastogi, D. K. Mitra, and A. Bhattacharya, "A novel implementation of bidirectional coupling circuit for broadband, high-voltage, powerline communications," in Proc. Asia-Pacific Conference on Communications, Oct. 2005, pp. 38-42, doi: 10.1109/APCC.2005.1554014.

[120] X. Y. Wang and X. Gao, "The typical designs of PLC network in MV distribution network," in Proc. IEEE International Symposium on
Power Line Communications and Its Applications, Mar. 2012, pp. 1923, doi: 10.1109/ISPLC.2012.6201332.

[121] T. Ku, C.-S. Chen, C. H. Lin, M. S. Kang, and H. J. Chuang, "Identification of customers served by distribution transformer using power line carrier technology," in Proc. IEEE Conference on Industrial Electronics and Applications, May 2009, pp. 3476-3481, doi: 10.1109/ICIEA.2009.5 138 852

[122] J. Anatory, N. Theethayi, M. Kissaka, N. Mvungi, and R. Thottappillil, "The effects of load impedance, line length, and branches in the BPLC: Transmission-lines analysis for medium-voltage channel," IEEE Trans. on Power Delivery, vol. 22, no. 4, pp. 2156-2162, Oct. 2007, doi: 10.1109/TPWRD.2007.905789.

[123] T. Ku, C.-S. Chen, C. H. Lin, M. S. Kang, and H. J. Chuang, "Identification of customers served by distribution transformer using power line carrier technology," in Proc. IEEE Conference on Industrial Electronics and Applications, May 2009, pp. 3476-3481, doi: 10.1109/ICIEA.2009.5 138852

[124] F. J. A. Andrade, C. A. G. Marques, T. R. Oliveira, F. P. V. Campos, E. J. de Oliveira, and M. V. Ribeiro, "Preliminary analysis of additive noise on outdoor and low voltage electric power grid in Brazil," in Proc. IEEE International Symposium on Power Line Communications and Its Applications, Mar. 2013, pp. 109-113, doi: 10.1109/ISPLC.2013.6525834

[125] A. A. M. Picorone, L. R. Amado, and M. V. Ribeiro, "Linear and periodically time-varying PLC channels estimation in the presence of impulsive noise," in Proc. IEEE International Symposium on Power Line Communications and Its Applications, Mar. 2010, pp. 255-260, doi: 10.1109/ISPLC.2010.5 479915.

[126] A. Kosonen, J. Ahola, and A. Pinomaa, "Analysis of channel characteristics for motor cable communication with inductive signal coupling," in Proc. IEEE International Symposium on Power Line Communications and Its Applications, Mar. 2010, pp. 72-77, doi: 10.1109/ISPLC.2010.5 479906.

[127] A. Kosonen and J. Ahola, "Comparison of signal coupling methods for power line communication between a motor and an inverter," in Proc. Lappeenranta University of Technology, Aug. 2010, pp. 431-440, doi: 10.1049/iet-epa.2009.0114.

[128] A. Pinomaa, J. Ahola, and A. Kosonen, "Power-line communicationbased network architecture for LVDC distribution system," in Proc. IEEE International Symposium on Power Line Communications and Its Applications, Apr. 2011, pp. 358-363, doi: 10.1109/ISPLC.2011.5764 422.

[129] F. Grassi, S. A. Pignari, and J. Wolf, "Channel characterization and emc assessment of a plc system for spacecraft dc differential power buses," IEEE Trans. on Electromagnetic Compatibility, vol. 53, no. 3, pp. 664-675, Aug. 2011, doi: 10.1109/TEMC.2011.2125967.

[130] A. Pinomaa, J. Ahola, A. Kosonen, and P. Nuutinen, "Applicability of narrowband power line communication in an LVDC distribution network," in Proc. IEEE International Symposium on Power Line Communications and its Applications, Mar. 2014, pp. 232-237, doi: 10.1109/ISPLC.2014.6812344.

[131] P. Nuutinen, A. Pinomaa, J. Ahola, and A. Kosonen, "Power line communication network for a customer-end AC grid in an LVDC distribution system," in Proc. IEEE International Conference on Smart Grid Communications, Nov. 2014, pp. 224-229, doi: 10.1109/SmartGridComm.2014.7 007650

[132] A. Kosonen, A. Pinomaa, J. Ahola, and P. Nuutinen, "Noise analysis of a power-line communication channel in an LVDC smart grid concept," in Proc. IEEE International Symposium on Power Line Communications and Its Applications, Mar. 2013, pp. 41-46, doi: 10.1109/ISPLC.2013.6525822.

[133] F. Versolatto and A. Tonello, "An MTL theory approach for the simulation of MIMO power-line communication channels," IEEE Trans. on Power Delivery, vol. 26, no. 3, pp. 1710-1717, Jul. 2011, doi: 10.1109/TPWRD.2011.2126608.

[134] A. Perez, A. M. Sanchez, J. R. Regue, M. Ribo, R. Aquilue, P. Rodriguez-Cepeda, and F. J. Pajares, "Circuital and modal characterization of the power-line network in the plc band," IEEE Trans. on Power Delivery, vol. 24, no. 3, pp. 1182-1189, Jul. 2009, doi: 10.1109/TPWRD.2009.2014278.

[135] T. Pang, P. So, K. See, and A. Kamarul, "Modeling and analysis of common-mode current propagation in broadband power-line communication networks," IEEE Trans. on Power Delivery, vol. 23, no. 1, pp. 171-179, Jan. 2008, doi: 10.1109/TPWRD.2007.911015.

[136] L. Berger, A. Schwager, P. Pagani, and P. D. Schneider, MIMO Power Line Communications: Narrow and Broadband Standards, Eletromag- 
netic Compatibility and Advanced Processing. Boca Raton: CRC Press, 2014.

[137] M. S. P. Facina, H. A. Latchman, H. V. Poor, and M. V. Ribeiro, "Cooperative in-home power line communication: Analyses based on a measurement campaign," IEEE Trans. on Communications, vol. 64, no. 2, pp. 778-789, Feb. 2016, doi: 10.1109/TCOMM.2015.2499744.

[138] A. Schwager, D. Schneider, W. Baschlin, A. Dilly, and J. Speidel, "MIMO PLC: Theory, measurements and system setup," in Proc. IEEE International Symposium on Power Line Communications and Its Applications, Apr. 2011, pp. 48-53, doi: 10.1109/ISPLC.2011.5 764447.

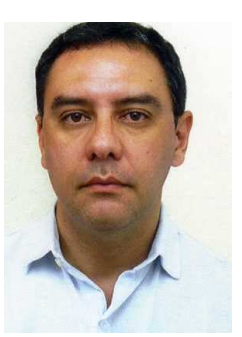

Luis G. S. Costa was born in Juiz de Fora MG, Brazil, in 1965. He has a Licentiate in Science (1992) and Post-Graduation in Telecommunications (1993) at Federal University of Juiz de Fora (UFJF). He received B.Sc. (2004) and M.Sc. (2012) degrees in Electrical Engineering from the UFJF, Brazil. Currently, he is a Ph.D. candidate at the same institution. Since 1984 he has been with Telemar Norte Leste (OI), where he is a supervisor in telecommunications. He has been a Professor at the Department of Electrical Engineering of the CES/JF since 2011. His main researchers involve digital communications, microwave engineering, and PLC coupling.

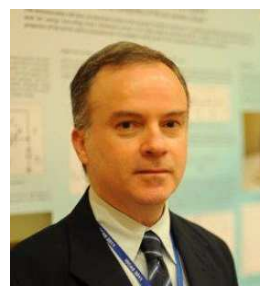

Antonio C. M. de Queiroz received the B.Sc. EE. Degree in 1979, the M.Sc. EE. Degree in 1984, and the D.Sc. EE. Degree in 1990 from the Universidade Federal do Rio de Janeiro, Rio de Janeiro, Brazil. Since 1982 he has been with the same institution, where he is now Titular Professor at the Department of Electronic Engineering and at the Electrical Engineering Program of the Coordenação dos Programas de Pós-graduação em Engenharia (COPPE). His main interests are in general circuit theory, filters and signal processing, computer-aided synthesis and analysis of circuits, energy harvesting using electrostatic generators, and the history of electricity.

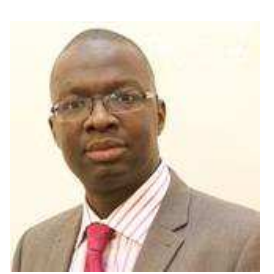

Adebisi Bamidele received his Master degree in advanced mobile communication engineering and $\mathrm{Ph} . \mathrm{D}$. in communication systems from Lancaster University, UK, in 2003 and 2009,respectively. Before that, he obtained a B.Sc. degree in Electrical Engineering from Ahmadu Bello University, Zaria, Nigeria, in 1999. He was a senior research associate in the School of Computing and Communication, Lancaster University between 2005 and 2012. He joined Manchester Metropolitan University, Manchester in 2012 where he is currently a Reader (Associate Professor) in Electrical and Electronic Engineering. He has worked on several commercial and government projects focusing on various aspects of wire-line and wireless communications. He is particularly interested in Research and Development of communication technologies for electrical energy monitoring/ management, transport, water, critical infrastructures protection, home automation, IoTs and Cyber-Physical Systems. He has several publications and a patent in the research area of data communications over power line networks and smart grid. He is a member of IET and a senior member of IEEE

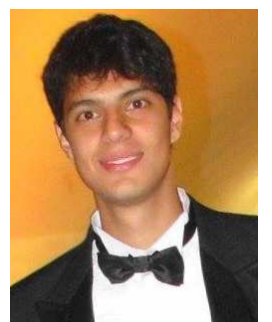

Vinicius L, R, da Costa was born in Juiz de Fora MG, Brazil, in 1992. He received the B.Sc. degree (2015) in Electrical Engineering from the Federal University of Juiz de Fora (UFJF), Brazil. Currently, he is an M.Sc. student at the same institution. Since 2014 has been with Smarti9 Ltda, where he is hardware/firmware programmer. His main interests are in general PLC theory, filters and signal processing, development of firmware, and hardware programming.

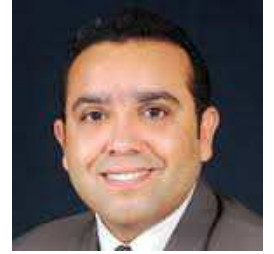

Moises V. Ribeiro received the B.S. degree in Electrical Engineering from the Federal University of Juiz de Fora (UFJF), MG, Brazil, and M.Sc. and D.Sc. degrees in Electrical Engineering from the University of Campinas, SP, Brazil, in 1999, 2001 and 2005, respectively. He was a Visiting Scholar at the University of California in Santa Barbara, CA, USA, in 2004, Visiting Professor (2005-2007) and Assistant Professor (2007-2015) at UFJF. Since 2015, he has been an Associate Professor at UFJF. He co-founded Smarti9 Ltd. and Wari Ltd. in 2012 and 2015, respectively. His research interests include signal processing, digital communication, power line communication, smart grids, the internet of things and smart city. In these fields, he has authored over 150 peer-reviewed papers, 9 book chapters, and filed 11 patents. He was the General Chair of the 2010 IEEE ISPLC, 2013 IWSGC, SBrT 2015, and a Guest Co-Editor for Special Issues in the EURASIP Journal on Advances in Signal Processing and EURASIP Journal of Electrical and Computer Engineering. He had served as the Secretary of the IEEE ComSoc TC-PLC. He was the recipient of Fulbright Visiting Professorship at Stanford University, Stanford, CA, USA, in 2011, and at Princeton University, Princeton, NJ, USA, in 2012. He was awarded Student Awards from 2001 IEEE IECON and 2003 IEEE ISIE. 